\title{
The functional outcome of direct lateral approach for fixation of proximal humeral fractures: A case series from a tertiary care hospital
}

Mujahid Jamil

Aga Khan University, mujahid.jamil@aku.edu

Irfan Anwer

Aga Khan University

Muhammed Tabish Saleem

Shaukat Khanum Memorial Cancer Hospital and Research Centre, Lahore, Pakistan

Ahmed Abdul Habib Khan

Shaukat Khanum Memorial Cancer Hospital and Research Centre, Lahore, Pakistan

Mohammad Zohaib Nawaz Khan

Shaukat Khanum Memorial Cancer Hospital and Research Centre, Lahore, Pakistan

Follow this and additional works at: https://ecommons.aku.edu/pakistan_fhs_mc_surg_orthop

Part of the Orthopedics Commons, and the Surgery Commons

\section{Recommended Citation}

Jamil, M., Anwer, I., Saleem, M. T., Habib Khan, A. A., Nawaz Khan, M. Z. (2019). The functional outcome of direct lateral approach for fixation of proximal humeral fractures: A case series from a tertiary care hospital. JPMA. The Journal of the Pakistan Medical Association, 69(12), 1915-1918.

Available at: https://ecommons.aku.edu/pakistan_fhs_mc_surg_orthop/130 


\section{The functional outcome of direct lateral approach for fixation of proximal humeral fractures: A case series from a tertiary care hospital}

Mujahid Jamil Khattak, ${ }^{1}$ Irfan Anwer, ${ }^{2}$ Muhammed Tabish Saleem, ${ }^{3}$ Ahmed Abdul Habib Khan, ${ }^{4}$ Mohammad Zohaib Nawaz Khan 5

\begin{abstract}
Displaced proximal humeral fractures warrant surgical fixation for early rehabilitation and better functional outcome. These fractures are traditionally fixed by delto pectoral surgical approach. Direct lateral approach has recently gained interest as it involves less soft tissue dissection and is particularly helpful in certain fracture patterns. However, there have been concerns of axillary nerve damage with this approach. We report a case series of proximal humerus fractures fixed by direct lateral approach from our institution. All displaced Type 2 and 3 fractures were included in our study. Pathological and comminuted Type 4 fractures and fractures with ipsilateral clavicle or elbow fractures were excluded. Oxford Shoulder Score was done at regular intervals for assessment of functional outcome. We did not observe any axillary nerve damage in our case series and the outcomes of $70.5 \%$ of our patients were excellent while in $29.5 \%$ it was good. We recommend direct lateral approach for specific pattern of proximal humerus fractures.
\end{abstract}

Keywords: Proximal humerus fracture, Direct lateral deltoid splitting approach.

\section{DOI:10.5455/JPMA.300885.}

\section{Introduction}

Proximal humeral fractures account for more than 50\% percent of all humeral fractures and $5-6 \%$ of all fractures in adults. ${ }^{1}$ Surgical management is indicated for displaced and comminuted proximal humerus fractures. Anatomical reduction and rigid fixation of these fractures results in quick rehabilitation and better functional outcome by minimising chances of muscle weakness and stiffness of shoulder inherently associated with this type of injury. ${ }^{2}$

Open reduction and fixation of proximal humeral fractures is performed commonly by the deltopectoral

1,2,4,5The Aga Khan University Hospital, Karachi, 3Shaukat Khanum Memorial Cancer Hospital and Research Centre, Lahore, Pakistan.

Correspondence: Mujahid Jamil Khattak. Email: mkhattak71@yahoo.com approach using Proximal Humerus Internal Locking System (PHILOS) plating. Although deltopectoral approach is generally favoured, extensive soft tissue dissection and displacement is required with this approach. Moreover, it is difficult to reduce and fix the posterolateral displaced, greater tuberosity fragment with deltopectoral approach; accurate reduction of this fragment is vital for optimal function of rotator cuff muscles.

Direct lateral Deltoid splitting approach helps in direct reduction of posterolateral displaced tuberosity fragments and is less time consuming as the plate can be applied easily. However, there has been a theoretical concern of damaging the axillary nerve with this approach. Also this approach is of less value in comminuted four part fractures because of only limited lateral exposure. Recently, Singh et al, however reported no incidence of nerve damage in a retrospective review of patients who underwent fixation by the lateral transdeltoid approach. ${ }^{3}$ Liu et $\mathrm{al}^{4}$ also found trans deltoid approach to be the better alternative in patients due to better function and range of motion achieved with this approach.

Our literature search does not show any study assessing the outcomes of proximal humerus fracture with trans deltoid approach from Pakistan. We report our series of patients with proximal humerus fractures who underwent fixation with direct lateral trans deltoid approach at the Aga Khan University Hospital, Karachi. The objective of our study is to assess the functional outcomes and morbidity associated with Direct Lateral trans-deltoid approach in proximal humerus fractures in our series.

\section{Case Series}

This is a retrospective study of 17 cases of proximal humerus fractures. The study period was from June 2015 to February 2018. According to Neer's Classification 5 based on standard AP and lateral $X$ rays, Type 2 and Type 3 proximal humerus fractures were identified and included for our study. Pathological fractures, comminuted 4 part fractures, fractures 
Table-1 (A): Patient demographics, fracture details and functional outcome.

\begin{tabular}{lcc}
\hline & Variable & $\mathrm{N}(\%)$ \\
\hline \multirow{2}{*}{ Gender } & Male & $08(47.0 \%)$ \\
Side & Female & $09(53.0 \%)$ \\
& Right & $06(35.3 \%)$ \\
Mechanism of Injury & Left & $10(58.8 \%)$ \\
& Bilateral & $01(5.9 \%)$ \\
Fracture Type & Injury due to FALL & $13(76.5 \%)$ \\
& Injury due to RTA & $02(11.8 \%)$ \\
OXFORD Shoulder Score & Injury due to Gun Shot & $02(11.8 \%)$ \\
& Neer TYPE 03 & $08(47.0 \%)$ \\
Anaesthesia & Neer TYPE 02 & $10(47.0 \%)$ \\
& Good & $05(29.5 \%)$ \\
Axillary nerve injury & Excellent & $12(70.5 \%)$ \\
\hline
\end{tabular}

Table-1 (B): Patient details and radiological outcome.

\begin{tabular}{lc}
\hline Variable & Mean \\
\hline Age (Years) & $57 \pm 12.5$ \\
Duration of Stay (Days) & $05 \pm 4.0$ \\
Duration of Surgery (Minutes) & $51.3 \pm 17.1$ \\
Radiological Union & $15.29 \pm 1.61$ \\
\hline
\end{tabular}

presenting with neurological deficit or associated shoulder dislocation at the time of presentation were excluded. Ethical approval from institutional Review Committee was obtained before commencement of our study.

Pre-operative anaesthetic fitness was obtained and prophylactic IV antibiotic (Cefazolin) was given at the time of surgery. Beach chair position was used for the procedure. After standard preparation and draping, lateral end of the Clavicle, Acromion, tip of the Coracoid and Deltoid tuberosity were identified and marked. A vertical incision was made at the junction of anterior one third and posterior two thirds of the acromion. The length of the incision varied according to fracture geometry, anticipated length of the plate and the arm circumference. Axillary nerve is usually located $5-7 \mathrm{~cm}$ distal from the lateral edge of the acromion. This area was secured with suture marker to avoid damage (Figure-1). Deltoid was then split in the line of its fibers. Attachments of the rotator cuff, greater tuberosity and the fracture fragments were identified. The greater tuberosity and the other fragments were gently reduced and stabilised temporarily with $\mathrm{K}$ wires. PHILOS plate was then applied laterally by sliding underneath the

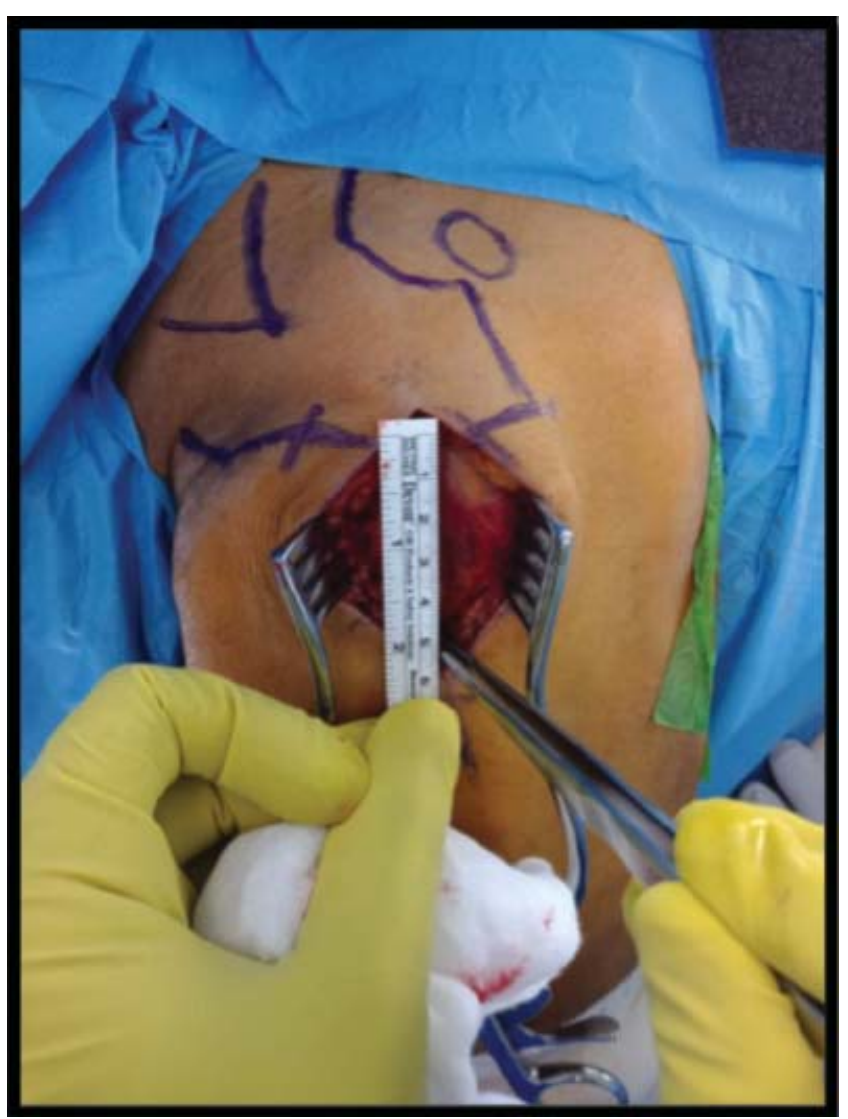

Figure-1: Patient in Beach-Chair position for right sided Humeral fracture, landmarks are identified, and after the incision, distance is measured from the acromion to protect the axillary nerve.

preserved deltoid fibers to avoid axillary nerve damage. Deltoid muscle was split again below the level of the axillary nerve. The upper and the lower windows, above and below the axillary nerve zone allows safe placement of the locking screws to secure the fracture with the plate. Fracture reduction and the plate position were checked with fluoroscopic assistance and the wound closure done in the routine fashion.

Cuff and collar was given post-operatively and shoulder exercises were started on the second postoperative day. Immediate follow up of patients was at one week for wound check and after 2 weeks for removal of stitches. Successive follow-ups were at 1 month, 3 months and 6 months respectively. Patients were evaluated for radiological union, axillary nerve dysfunction, and shoulder function. Functional assessment was done using OXFORD shoulder score. ${ }^{6}$ The results were graded as Excellent, Good, Fair and Poor.

A total of 17 patients were included in the study of which 
$8(47.0 \%)$ patients were male and $9(53.0 \%)$ were females. Mean age was $57 \pm 12.5$ years. Right side was involved in 6 patients and left side in 11 (70.5\%) patients. There was one case of bilateral proximal humerus fracture. Twelve of our patients sustained injury due to a fall, one $(5.8 \%)$ patient had history of road traffic accident, 2 (11.7\%) patients had history of gunshot injury involving penetrating abdominal wounds along with humeral fracture and underwent laparotomy.

Among the type of fractures, 8 (47.0\%) were Neer's Type 3 and 9 (47.0\%) fractures were identified as Neer's Type 2. Average time duration of surgery was $51.3 \pm 17.1$ minutes and average length of stay at hospital was $5 \pm 4.0$ days, except for the 2 cases suffering from gunshot injury, their length of stay was 11 and 18 days respectively. Two $(11.7 \%)$ patients were operated under regional anaesthesia while 15 (88.2\%) patients including one case of bilateral humerus fracture were operated under general anaesthesia. There was no axillary nerve dysfunction noted in any of the patients and according to OXFORD Shoulder Score - 12 (70.5\%) patients showed excellent results while 5 (29.5\%) patients, including those with bilateral fractures showed good results. The mean follow up was $22 \pm 10$ weeks. Radiological union time mean was $15 \pm 2$ weeks. There was no case of non-union, infection or varus malunion and/or acromion impingement.

\section{Discussion}

Deltopectoral approach is commonly used for majority of the surgeries around the shoulder including, fixation of proximal humeral fractures. Surgeons prefer this approach because of its familiarity and common use. This approach is however cumbersome and time consuming and is associated with extensive soft tissue retraction, especially in comminuted fractures of the proximal humerus. It is technically difficult to reduce posterolateral displaced tuberosity fragment by anterior delto pectoral approach. Moreover, the PHILOS plate used for fixation of proximal humerus fracture is usually applied laterally and application of the plate becomes a challenge especially in obese or younger patients with good muscle mass. Direct lateral approach has recently gained attention for the fixation of such injuries. The main concern with deltoid splitting approach is axillary nerve damage. The axillary nerve traverses the deltoid muscle 4-6 cm below the tip of the acromion. This area must be identified and protected during the procedure. ${ }^{7-9}$

Recent studies of the direct lateral deltoid approach have shown excellent results for such fractures with a good outcome and in decrease in time required for the surgery. Gardner et al 10 operated on 16 patients with this approach and found it very useful and safe. Isiklar et al11 found better results with deltoid splitting approach as compared to deltopectoral approach with no axillary nerve damage. Robinson et al reported a series of 386 humeral fractures that were fixed with direct lateral deltoid splitting approach. They have added some interesting variations to the approach based on the fracture pattern and extensions. ${ }^{12}$

Our study also demonstrated excellent and good functional outcomes in our patients as calculated by Oxford Shoulder Score, with no post-operative axillary nerve palsy or deltoid muscle dysfunction and excellent radiological outcome. However, direct lateral approach only gives limited and lateral exposure and therefore not suitable for 4 part fractures with extensive comminution.

\section{Conclusion}

We can conclude that direct lateral deltoid splitting approach is a good alternative in selected cases of proximal humeral fractures without any significantly adverse clinical effects. A larger sample size with a longer duration of follow up is required for comparison with the traditional deltopectoral approach in relation to functional outcome.

Disclaimer: The study has been presented in surgical grand round at the Aga Khan University Hospital in 2017.

Conflict of Interest: The authors declare that they have no conflict of interest.

Funding Disclosure: No funding was made available for this study.

Consent: Verbal consent has been taken from all of our patients for the publication of this case report.

\section{References}

1. Neer CS II, Rockwood CA. Fractures and dislocations of the shoulder, in Rockwood and Green: Fractures in adults. Philadelphia: Lippincott, 1984; pp 675-721.

2. Nho SJ, Brophy RH, Barker JU, Cornell C, MacGillivray JD. Management of proximal humeral fractures based on current literature. J Bone Joint Surg Am. 2007; 89:44-58.

3. Singh, Harpreet, AshishBatra, Dhaval Patel. "Lateral transdeltoid approach to proximal humerus fractures." Inter Surg J. 2015; 2:337-40.

4. Liu K, Liu PC, Liu R, Wu X. Advantage of minimally invasive lateral approach relative to conventional deltopectoral approach for treatment of proximal humerus fractures. Med Sci Monit. 2015; 21:496-504.

5. Neer CS. Displaced proximal humeral fractures. J Bone Joint Surg. 1970; 52:1090-103. 
6. Kirkley A, Griffin S, Dainty K. Scoring systems for the functional assessment of the shoulder. Arthroscopy. 2003; 19:1109-20.

7. Cetik O, Uslu M, Acar HI, Comert A, Tekdemir I, Cift H. Is there a safe area for the axillary nerve in the deltoid muscle? a cadaveric study. J Bone Joint Surg Am. 2006; 88:2395-9.

8. Burkhead WZ Jr, Scheinberg RR, Box G. Surgical anatomy of the axillary nerve. J Shoulder Elbow Surg. 1992; 1:31-6.

9. Robinson CM, Khan L, Akhtar A, Whittaker R. The extended deltoid-splitting approach to the proximal humerus. J Orthop Trauma. 2007; 21:657-62.

10. Gardner MJ, Griffith MH, Dines JS, Briggs SM, Weiland AJ, Lorich
DG. The extended anterolateral acromial approach allows minimally invasive access to the proximal humerus. Clin Orthop Relat Res. 2005; 123-9.

11. Isiklar Z, Kormaz F, Gogus A, Kara A. Comparison of deltopectoral versus lateral deltoid split approach in operative treatment of proximal humeral fractures. Orthopaedic Proceedings. 2018; 92: SUPP II.

12. Robinson CM, Murray IR. The extended deltoid-splitting approach to the proximal humerus, Variations and extensions. J Bone Joint Surg Br. 2011; 93:387-92. 